Rev. Biol. Trop., 48(2/3): 465-472, 2000

www.ucr.ac.cr www.ots.ac.cr www.ots.duke.edu

\title{
Nueva especie de Ilyodon (Cyprinodontiformes: Goodeidae) de la cuenca del río Balsas, México
}

Joel Paulo-Maya ${ }^{1}$ y Patricia Trujillo-Jiménez ${ }^{2}$

1 Lab. de Ictiología y Limnología, Esc. Nac. Cienc. Biol. I.P.N. Carpio y Plan de Ayala s.n. Código Postal 11340 México, D.F. Fax (01) 5729-60-00 ext.62420 jpaulo@vmredipn.ipn.mx.

2 Lab. Ictiología, Centro de Investigaciones Biológicas, U.A.E.M. Av. Universidad 1001 Col. Chamilpa, Código Postal 62210 Cuernavaca, Morelos. México. Fax (01 - 7) 329-70-56 trujillo@ cib.uaem.mx

\section{Recibido 26-II-1999. Corregido 28-II-2000. Aceptado 8-III-2000}

\begin{abstract}
A new species of the fish genus Ilyodon is described from San Juan Tacámbaro river, Michoacán, México $19^{\circ} 10^{\prime} 42^{\prime \prime} \mathrm{N}, 101^{\circ} 20^{\prime} 45^{\prime \prime} \mathrm{W}$. We used 129 specimens representing I. whitei, 60 of I. furcidens and 84 of the new species for our analyses. These included samples of both sexes. The new species is distinguished from I. whitei and I. furcidens by its strongly convex cephalic profile, the size and disposition of the pores of the lateral line of the preorbital, the thinner caudal peduncle, its strongly bifid tooth and the form and size of neural prezygapophyses of caudal vertebrae.
\end{abstract}

Key words: New species, Goodeidae, Ilyodon, San Juan Tacámbaro, Balsas basin, México.

La familia Goodeidae en México está representada por aproximadamente 42 especies de peces vivíparos (Mayden et al. 1992). La mayoría de los miembros de la familia viven en las partes altas del Altiplano Central Mexicano y ocupan una variedad de hábitats; sin embargo, algunos géneros como Goodea, Ataenobious, Xenoophorus y Xenotoca habitan cuencas de la vertiente Atlántica y otros como Allodontichthys, Ameca, Neoophorus, Skiffia e Ilyodon en las cuencas de la vertiente del Pacifico (Miller 1986).

Desde la revisión de la familia por Hubbs y Turner (1939), los conocimientos científicos posteriores han conducido a numerosos cambios, los cuales aun se siguen presentando. Para el género Ilyodon hay varios estudios taxonómicos encaminados a dilucidar el número de especies que lo conforman, utilizando para ello métodos electroforéticos, cariotipos y cruzas controladas (Turner y Grosse 1980, Grudzien y Turner 1984). A la fecha estos trabajos han permitido reconocer solo dos especies: Ilyodon furci- dens (Jordan y Gilbert) 1882 o mexcalpique del Ameca e Ilyodon whitei (Meek) 1904 o mexcalpique del Balsas. En el presente trabajo se presentan evidencias de la existencia de una nueva especie para el género.

\section{MATERIALES Y MÉTODOS}

A los ejemplares analizados se les tomaron las medidas proporcionales y las cuentas merísticas como indica Miller (1948). Los poros de la línea lateral cefálica fueron contados de acuerdo a Gosline (1949). El radio anal anterior rudimentario fue incluido en la cuenta de la aleta anal. Los dos últimos radios de las aletas dorsal y anal fueron contados como un radio. Para los datos merísticos el número de especímenes considerados es dado entre paréntesis y las cuentas correspondientes al holotipo se indican con un asterisco. Para el estudio se utilizaron 129 adultos de $I$. whitei (IPN-P4764) del río Amacuzac en el poblado 
Chisco, Morelos; P. Trujillo-Jiménez, 13 de junio de 1995. 60 adultos de I. furcidens (IPN-P1946), río Tamazula, Jalisco; J. Alvarez, 9 de marzo de 1956 y 82 adultos de la nueva especie (IPNP4380), manantiales de Cutzaróndiro en San Juan Tacámbaro, Michoacán; A. Ramírez et al., 10 de mayo de 1986. Las muestras incluyen ambos sexos. Todos los especímenes fueron catalogados en la Colección de Peces Dulceacuícolas Mexicanos de la Escuela Nacional de Ciencias Biológicas (IIPN). El acrónimo institucional sigue a Leviton et al. (1985).

Para discernir la forma y tamaño de las distintas piezas óseas, así como la disposición de los poros cefálicos de la línea lateral, se transparentaron cinco hembras y cinco machos de cada una de las especies.

En la comparación morfológica intragenérica se elaboró un análisis morfométrico multivariado siguiendo los pasos de Rauchenberger (1988), para lo cual se realizó un diagrama de entramado sobre esquemas de las tres especies, usando los siguientes puntos de anclaje (Fig. 1): 1) hocico; 2) occipucio; 3,4) base de la aleta dorsal; 5,6) placa hipúrica; 7,8) base de la aleta anal; 9) origen de las aletas pélvicas y 10) sínfisis cleitral. Estos puntos permiten establecer una cobertura que es utilizada para la comparación de las formas (Humphries et al. 1981). Todas las medidas fueron tomadas con un vernier digital con una precisión de $0.01 \mathrm{~mm}$. La comparación morfológica se obtuvo del Análisis de Componentes Principales de los logaritmos de las medidas antes mencionadas. Todos los análisis se realizaron con el programa ANACOM Versión 3.0 (De la Cruz 1994).

Para cuantificar las diferencias morfométricas entre sexos de la nueva especie procedimos a usar las medidas proporcionales comúnmente consideradas.

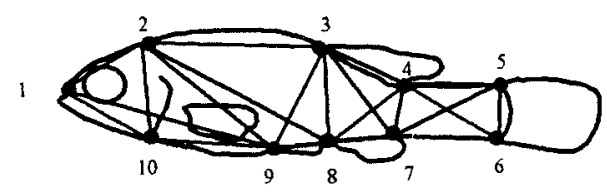

Fig. 1. Entramado de medidas utilizado para las especies de Ilyodon. Los puntos numerados representan los nodos de anclaje; las distancias definidas por estos puntos fueron utilizados en el ACP

Fig. 1. Truss network to species of Ilyodon. Numbered points represent landmarks; interlandmark distances defined by these points were used in PCA

\section{Ilyodon cortesae sp. nov. Mexcalpique del Tacámbaro}

(Figs. 2 y 3 )

Holotipo: IPN-P4380; macho adulto de $46.1 \mathrm{~mm}$ de longitud estándar (LS), recolectado por A. Ramírez, J. Paulo-Maya, P. Vallejo y H. Ríos. 10 de mayo de 1986. Manantiales de Cutzaróndiro, $13.1 \mathrm{~km}$. al suroeste del río San Juan Tacámbaro $\left(19^{\circ} 10^{\prime} 42^{\prime \prime} \mathrm{N}, 101^{\circ} 20^{\prime} 45^{\prime \prime}\right.$ W) (Fig. 4). La localidad se encuentra a 1463 metros sobre el nivel del mar.
Fig. 2. Ilyodon cortesae IPN -P4381: arriba, Holotipo, macho $46.1 \mathrm{~mm}$ LS; abajo,
paratipo, hembra $54.24 \mathrm{~mm}$ LS

Fig. 2. Ilyodon cortesae, IPN-P4381: above, Holotype, male, $46.1 \mathrm{~mm}$ LS; below, paratype, female, $54.24 \mathrm{~mm} \mathrm{LS}$. 
A

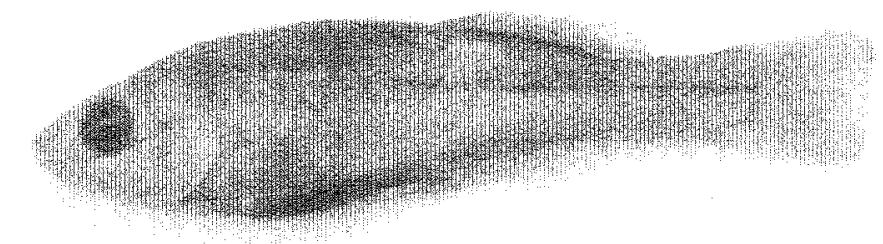

B

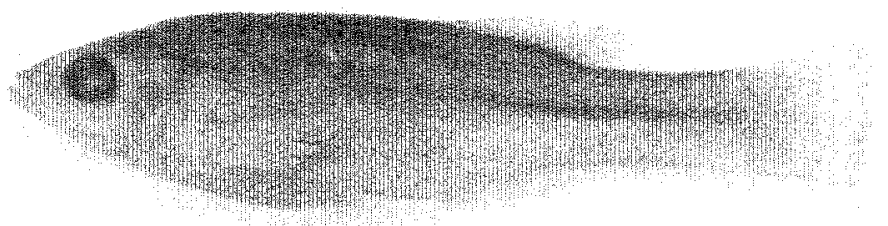

$\mathbb{C}$

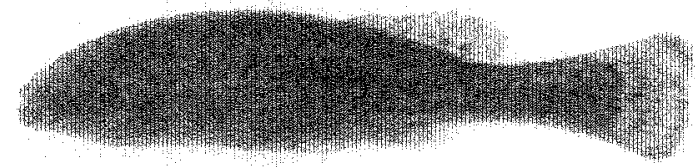

Fig. 3. Especies del género Ilyodon a) I. furcidens b) I. whitei c) I. cortesae

Fig. 3. Species of genus Ilyodon a) I. furcidens b) I. whitei c) I. cortesae

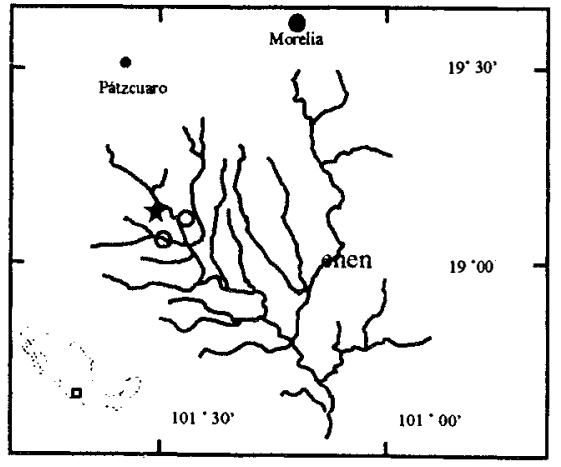

Fig. 4. Mapa de distribución de Ilyodon cortesae. Localidad Tipo $=$ estrella

Fig. 4. Distribution map of Ilyodon cortesae. Type Locality $=$ Star.

Paratipos: IPN-P4380; 81 especímenes: 36 machos y 45 hembras recolectados con el holotipo. El intervalo de longitud estándar observado va de 32.42 a $51.4 \mathrm{~mm}$. UANL-14214; 1 macho y una hembra recolectados con el holotipo
Diagnosis: Es la más pequeña de las especies de Ilyodon, con una longitud estándar máxima observada de $51.5 \mathrm{~mm}$. El perfil cefálico es convexo, contrario a lo que se presenta en las otras especies, donde es más bien recto (Figs. 2, 3 y 5). La base del pedúnculo caudal es la más angosta en el género. Presenta de 52 a 60 escamas en una serie longitudinal (Cuadro 1). Tiene dos series irregulares de dientes fuertemente bífidos. Los poros de la línea lateral en la rama preorbital son más amplios y siempre dispuestos en línea recta. Las precigapófisis de las vértebras del pedúnculo caudal son muy cortas y no tocan a las postcigapófisis adyacentes.

Descripción: Los aspectos generales de la morfología y pigmentación son notorios en las Figs. 2 y 3. Los datos morfológicos están dados en el Cuadro 2. El cuerpo es comprimido y grácil, con las aletas medias situadas ligeramente por detrás de la mitad del cuerpo. 
El origen de la aleta dorsal es ligeramente anterior al origen de la aleta anal. En alcohol etílico el cuerpo tiene el dorso café obscuro y el vientre más claro. Los lados tienen una línea negra de pequeños puntos que van desde el fin del opérculo hasta la base de la aleta anal. Nu- merosas manchas negras se encuentran en todo el cuerpo e incrementan su densidad en la porción posterior del pez. La aleta dorsal del macho muestra tres series longitudinales de puntos negros. Las aletas pectorales y pélvicas son ligeramente más obscuras.

\section{CUADRO 1}

Distribución de frecuencias de las escamas en una serie longitudinal para especies de Ilyodon

Frequency distribution of lateral line scales for species of Ilyodon.

Número de escamas en una serie longitudinal

Especies
I. furcidens
I. whitei
I. cortesae

4344
$\begin{array}{lllllllllllllllll}44 & 45 & 46 & 47 & 48 & 49 & 50 & 51 & 52 & 53 & 54 & 55 & 56 & 57 & 58 & 59 & 60\end{array}$
$\begin{array}{llllllll}2 & 2 & 5 & 4 & 9 & 3 & 1 & 2\end{array}$
$\begin{array}{lllllllll}2 & 3 & 2 & 5 & 14 & 13 & 8 & 10 & 6\end{array}$
$\begin{array}{lllllllll}6 & 7 & 13 & 17 & 13 & 18 & 1 & 1 & 1\end{array}$

\section{CUADRO 2}

Medidas proporcionales de adultos de Ilyodon cortesae, expresadas en milésimas de LS. Basados en IPN P4381. Los datos incluyen al holotipo.

Proportional measurements of adults of llyodon cortesae, expressed thousandths of SL. Based on IPN P4381. Data includes holotype.

Medidas

Longitud estándar (mm)

Distancia predorsal

Distancia prepélvica

Base anal a origen caudal

Altura máxima

Anchura máxima

Longitud cefálica

Altura cefálica

Anchura cefálica

L. del pedúnculo caudal

Altura mínima

Distancia interorbital

Hocico

Diámetro ocular

Amplitud boca

Longitud mandibular

Base de la dorsal

Dorsal deprimida

Base de la anal

Anal deprimida

Longitud de la caudal

Longitud de la pectoral

Longitud de la pélvica

$\begin{array}{cc}\text { Holotipo } & \text { Intervalo (38) } \\ & \\ 46.09 & 32.42-51.40 \\ 603 & 583-642 \\ 485 & 358-545 \\ 310 & 305-491 \\ 276 & 238-300 \\ 157 & 147-208 \\ 251 & 217-292 \\ 200 & 173-255 \\ 156 & 148-189 \\ 246 & 224-290 \\ 97 & 88-117 \\ 116 & 98-139 \\ 65 & 64-101 \\ 94 & 62-100 \\ 83 & 77-132 \\ 49 & 40-70 \\ 187 & 138-192 \\ 238 & 157-272 \\ 67 & 55-97 \\ 110 & 103-153 \\ 198 & 188-240 \\ 170 & 130-175 \\ 124 & 97-134\end{array}$

Machos $\chi$
42.33
615
505
349
267
177
264
201
173
265
106
119
82
87
104
53
156
211
78
135
215
152
117

Intervalo (46)

$\begin{array}{cc}34.87-51.0 & 41.1 \\ 568-631 & 599 \\ 465-535 & 499 \\ 280-380 & 339 \\ 240-291 & 265 \\ 157-203 & 173 \\ 170-297 & 267 \\ 193-233 & 207 \\ 117-185 & 169 \\ 119-348 & 272 \\ 69-125 & 105 \\ 81-133 & 117 \\ 61-105 & 83 \\ 67-99 & 89 \\ 81-171 & 103 \\ 41-80 & 56 \\ 167-233 & 196 \\ 82-317 & 269 \\ 44-94 & 66 \\ 85-144 & 116 \\ 199-284 & 231 \\ 135-184 & 163 \\ 111-137 & 125\end{array}$




\section{ENGLISH DESCRIPTION}

Diagnosis: The new species is the smallest Ilyodon, with a maximum standard length of $51.5 \mathrm{~mm}$. The head profile is more convex than in the other species of the genus. The caudal peduncle is the thinnest within Ilyodon. It has 52 to 60 scales in a longitudinal series (Table 1). The jaw has two irregular series of strongly implanted bifid teeth. The pores of the lateral line in the preorbital branch are wide and always in a straight line. The prezigapophyses of caudal peduncle vertebrae are short and do not touch the adjacent postzigapophyses (Fig. 4).

Description: Gereral morphology and pigmentation appear in Figs. 2 and 3 and morphological data in Table 2. The body is compressed and graceful, with the medial fins situated slightly to the back of the mid-body. The dorsal fin originates slightly a head of the anal fin. In ethylic alcohol the back is dark brown (with a clearer belly). The sides have a black line of small dots from the end of the operculum to the anal fin base. Numerous black marks cover the body and concentrate in the posterior portion of the fish. The dorsal fin of the male has three longitudinal series of black dots. The pectoral and pelvic fins are lightly darker.

Datos merísticos: Escamas en una serie longitudinal 52(6), 53*(7), 54(13), 55(17), 56(13) 57(18), 58(8), 59(1), 60(1); radios de la aleta caudal 22(2), 23(13), 24*(19), 25(21), 26(22), 27(6); radios de la aleta pectoral 12(2), $13 *(82)$; radios de las aletas pélvicas (ambas): $6 *(84)$; radios de la aleta anal: 11(4), 12*(59), 13(21); radios de la aleta dorsal: 15(8), $16 *(48), 17(25), 18(3)$; branquiespinas: $23(2)$, 24(5), 25(1), 26(0), 27*(8), 28(7), 29(0), 30(4), $31(3), 32(5)$. Poros en la línea lateral cefálica: mandibular 2(23), $3 *(53)$; preorbital 4(23), $5 *(53)$; preopercular $6(2), 7 *(63), 8(11)$; supraorbital, canales discontinuos entre los poros 2A y 2B (Tipo II de Gosline 1949), 6(3), $7 *(29), 8(28), 9(16)$ considerando el canal izquierdo; 6(8), $7 *(16), 8(30), 9(21), 10(1)$ considerando el canal derecho. (El número con asterisco corresponde al Holotipo).
Dientes: Si bien el número y disposición de los dientes, así como la forma y posición de la boca, no pueden ser considerados como un aspecto primario en el reconocimiento de las distintas especies de Ilyodon (Turner y Grosse 1980, Grudzien y Turner 1984), es notable la diferencia existente en las tres especies respecto a la profundidad de la incisión en los dientes bífidos, pues para los ejemplares de I. cortesae ésta abarca aproximadamente un tercio del tamaño de la pieza y en ejemplares de las otras especies siempre llega como máximo hasta un sexto de su dimensión total (Fig. 5a) (Student p < 0.05).

Rama preorbital de la línea lateral: Los poros de I. cortesae son distintos a los conocidos para el género, pues además de ser el doble de anchos, estos se arreglan longitudinalmente a lo largo de la rama; caso contrario para I. furcidens e I. whitei quienes presentan poros mas reducidos y dispuestos de manera alterna (Fig. 5b).

Vértebras caudales: Las tres especies consideradas difieren notablemente en tamaño y forma de las apófisis de las vértebras caudales, pues como puede observarse en la Fig. 5c, las apófisis vertebrales de I. furcidens se ponen siempre en contacto unas con otras, caso distinto en I. whitei que no presenta postcigapófisis debido a que las espinas neurales se ubican muy retrasadas en el cuerpo vertebral y son sostenidas en parte por las precigapófisis bicéfalas de la vértebra posterior adyacente. Por el contrario en I. cortesae nunca se ponen en contacto las apófisis vertebrales y las espinas neurales nacen a la altura media del cuerpo vertebral.

Análisis multivariado: El primer componente (CPI) explica el $91.6 \%$ de la varianza total (Cuadro 3). Las altas cargas positivas asociadas con este componente sugieren que representa el factor general de tamaño. El segundo (CPII) y tercer componente(CP III) parecen no confundir las diferencias de tamaño porque los puntos son paralelos a PCI, por lo que no fue necesario aplicar el método de corrección de Humphries et al. (1981) (Rauchenberger 1988). El PC II explica el 2. $08 \%$ de la varianza total y el PC III el $1.43 \%$. 


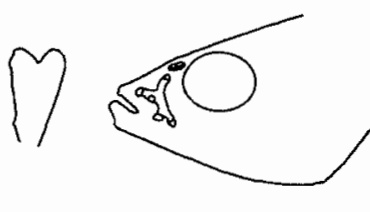

I. furcidens

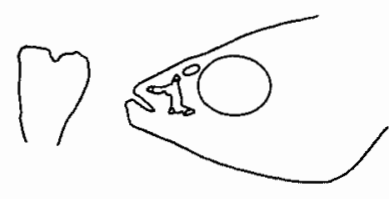

I. whitei

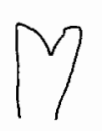

$\mathbf{a}$

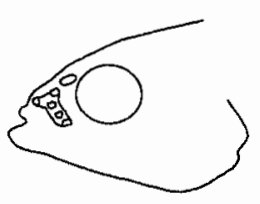

b

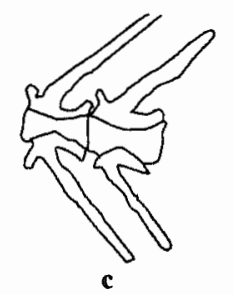

I. cortesae
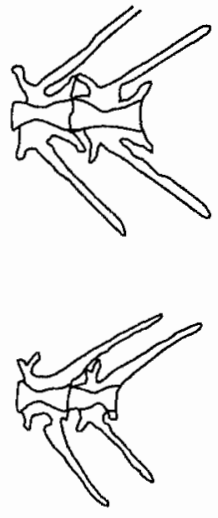

\section{CUADRO 3}

Coeficientes del análisis de componentes principales de las variables morfométricas entre especies de Ilyodon

Coefficients from principal components analysis of morphometric variables among species of Ilyodon

$\begin{array}{cccc}\begin{array}{c}\text { Variables } \\ \text { \% Varianza }\end{array} & \text { PC I } & \text { PC II } & \text { PC III } \\ & 91.6 & 2.08 & 1.43 \\ 1-10 & 0.18 & -0.14 & 0.09 \\ 1-9 & 0.22 & -0.13 & -0.09 \\ 1-2 & 0.15 & -0.32 & 0.33 \\ 2-10 & 0.20 & -0.20 & 0.15 \\ 2-9 & 0.25 & -0.17 & -0.16 \\ 2-8 & 0.27 & -0.05 & -0.09 \\ 2-3 & 0.27 & -0.11 & -0.32 \\ 3-9 & 0.23 & -0.09 & -0.15 \\ 3-8 & 0.22 & 0.18 & 0.12 \\ 3-7 & 0.22 & 0.19 & 0.10 \\ 3-4 & 0.22 & 0.24 & 0.70 \\ 4-8 & 0.20 & 0.35 & -0.03 \\ 4-7 & 0.19 & 0.30 & -0.24 \\ 4-6 & 0.23 & -0.12 & -0.15 \\ 4-5 & 0.22 & -0.22 & -0.05 \\ 5-7 & 0.24 & -0.003 & 0.10 \\ 5-6 & 0.18 & 0.30 & -0.006 \\ 6-7 & 0.25 & -0.11 & 0.15 \\ 7-8 & 0.11 & 0.31 & -0.18 \\ 8-9 & 0.24 & -0.001 & 0.06 \\ 9-10 & 0.24 & -0.08 & -0.20\end{array}$

Fig. 5. Caracteres diagnósticos de Ilyodon a) dientes b) línea lateral preorbital c) vértebras caudales

Fig. 5. Diagnostic Characters of Ilyodon a) Tooth b) preorbital lateral line c) caudal vertebrates

I. cortesae se separa de las otras dos especies sobre el CP II, como se puede observar en la Fig. 6. Los valores en este componente indican que I. cortesae posee las mas pequeñas distancias en las dimensiones de la aleta dorsal al origen y fin de la aleta anal (segmentos 4-7 y 4-8). Esto sugiere que la base del pedúnculo caudal de la nueva especie es el más angosto dentro del género.

Dimorfismo sexual: Los sexos de I. cortesae claramente difieren en su tamaño; las hembras en general son más grandes que los machos (Student $\mathrm{p}<0.05$ ). Al mismo tiempo, la base de la aleta anal, así como su longitud cuando está deprimida, es más grande en las hembras (Student $\mathrm{p}<.0 .0001$ ). Sin embargo, la base de aleta dorsal y su longitud cuando está deprimida es mayor en los machos que en las hembras (Student $\mathrm{p}<0.002$ ). (Fig. 7).

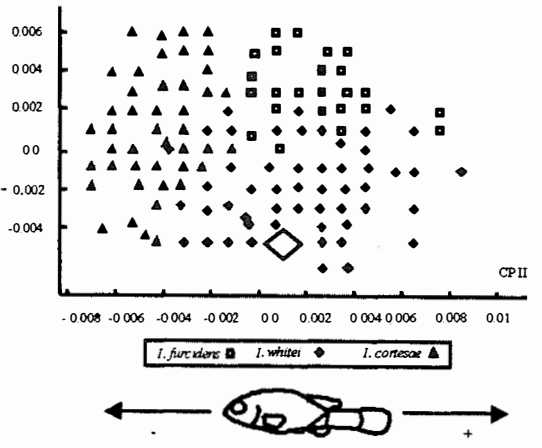

Fig. 6. Análisis de Componentes Principales : Comparación entre las especies del género Ilyodon

Fig. 6. Principal Component Analysis: Comparison between three species of Ilyodon. 

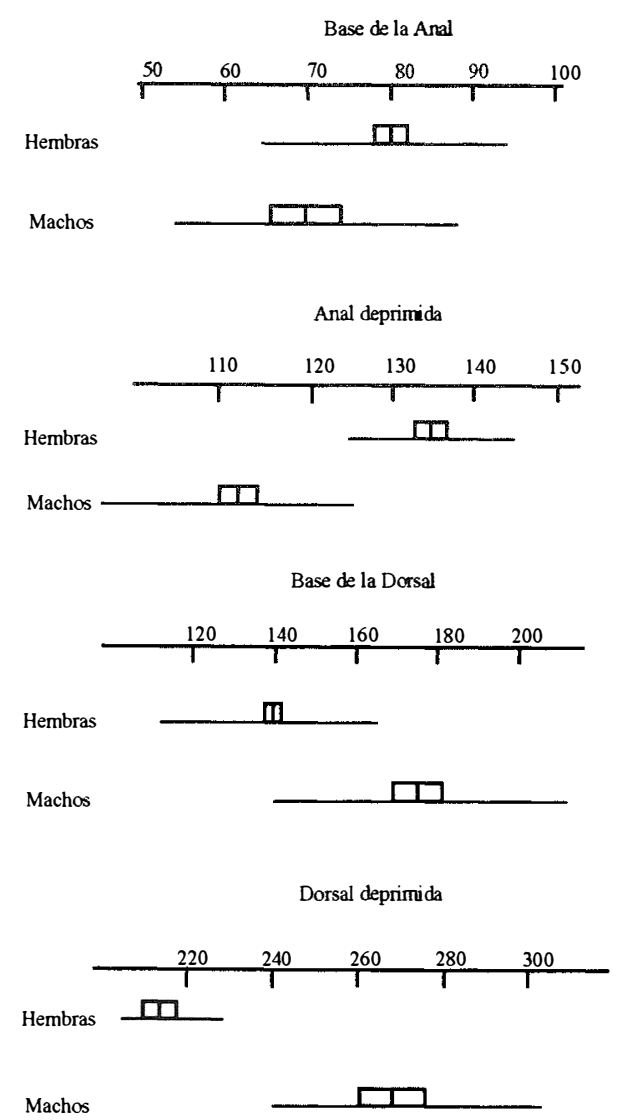

Fig. 7. Comparación de sexos de la nueva especie

Fig. 7. Comparison of sexes for the new species

Discusión: Es conocido que la taxonomía del género Ilyodon ha cambiado debido al reconocimiento en los ríos del sistema Armeria-Cohuayana de morfotipos "tróficos" de I. furcidens, que se distinguen en la forma y tamaño de la boca, así como en la morfología y disposición de sus dientes, morfotipos que eran considerados como especies diferentes (Turner y Grosse 1980, Grudzien y Turner 1984). Este hecho ha llevado a reconsiderar la variación de las especies de $\mathrm{Il}$ yodon a lo largo de su área de distribución.

Este fenómeno no se presenta para I. cortesae n. e. y I. whitei pues si bien comparten los rasgos propios del género, así como su área de distribución, existen rasgos distintivos en la nueva especie que no involucran los caracteres detectados para los morfotipos de I. furcidens como el mayor número de escamas en una serie longitudinal, el perfil cefálico tan convexo, la forma y disposición de los poros de la línea lateral en la rama preorbital, la anchura del pedúnculo caudal y la forma y tamaño de las apófisis en las vértebras caudales.

Distribución: Se conoce solo en manantiales y parte alta del río San Juan Tacámbaro, Michoacán.

Hábitat: La localidad tipo de I. cortesae corresponde a un manantial de aguas transparentes de 4 a $6 \mathrm{~m}$ de amplitud y 1 metro de profundidad. El fondo del cuerpo de agua es arenoso con zonas de grava y manchones de Potamogeton sp. y algas filamentosas. El agua tuvo $7.2 \mathrm{mg} / \mathrm{l}$ de oxigeno disuelto, un $\mathrm{pH}$ de 7.5 y una temperatura de $20^{\circ} \mathrm{C}$. $\mathrm{Hy}$ bopsis boucardi (Günther) 1868, familia Cyprinidae, es la única especie que cohabita en la localidad.

Etimología: El nombre de la especie se establece como una distinción a María Teresa Cortés, quien ha contribuido de manera sobresaliente al desarrollo de la Ictiología Mexicana.

\section{AGRADECIMIENTOS}

Agradecemos a Eugenia López López, O. Polaco, Edmundo Díaz Pardo, Aquiles Argote y Bruce G. Stewart por sus sugerencias al trabajo.

\section{RESUMEN}

Una nueva especie, Ilyodon cortesae, se describe del río San Juan Tacámbaro, Michoacán, México. Usamos 129 organismos pertenecientes a I. whitei, 60 de I. furcidens y 84 de la nueva especie para nuestro análisis. Estos incluyeron muestras de ambos sexos. La nueva especie se distingue de I. whitei y de I. furcidens por su perfil cefálico convexo, el tamaño y disposición de los poros de la línea lateral en el preorbital, la base del pedúnculo caudal delgado, sus dientes fuertemente bífidos y en la forma de las precigapófisis de las vértebras caudales. 


\section{REFERENCIAS}

De La Cruz, G. 1994. Sistema para el Análisis de Comunidades. ANACOM. Versión 3.0. Dep. Pes. y Biol Mar. Centro Interdisciplinario de Ciencias MarinasInstituto Politécnico Nacional México. 99 p.

Grudzien, T. A. \& B. J. Turner. 1984. Genic identity geographic differentation of trophically dichotomus $\mathrm{Il}$ yodon (Teleostei: Goodeidae). Copeia.: 102-107.

Hubbs, C.L. \& C.L. Turner. 1939. Studies of the fishes of the order Cyprinodontes. XVI. A revision of the Goodeidae. Misc. Pub. Mus. Zool. Univ. Michigan. 42.79 p.

Humphries, J. M., F. L. Bookstein, B. Chernoff, G. R. Smith, R. L. Elder \& S. G. Poss. 1981. Multivariate discrimination by shape in relation to size. Syst. Zool. 30: 291-308.

Leviton, A. E., R.H. Gibbs, Jr., E. Heal \& C. E. Dawson. 1985. Standards in ichthyology and herpetology: Part I. standard symbolic codes for institutional resource collections in herpetology and ichthyology. Copeia: 802-832.
Mayden R. L., M. B. Brooks, L M. Page \& R. R. Miller. 1992. The native freshwater fishes of North America p. 827-863. In R. L. Mayden (ed.) Systematic, Historical Ecology, \& North American freshwater fishes. Stanford, California.

Miller, R.R. 1948. The cyprinodont fishes of the Death Valley system of eastern California and southwestern Nevada. Misc. Pap. Mus. Zool. Univ. Michigan 68: 1-155

Miller, R. R. 1986. Composition and derivation of the freshwater fish fauna of Mexico. Anal. Esc. Nal. Cien. Biol., Méx. 30: 121-153.

Rauchenberger, M. 1988. A new species of Allodontichthys (Cyprinodontiformes: Goodeidae), with comparative morphometrics for the genus. Copeia.: 433-441.

Turner, B.J. \& D. J. Grosse. 1980. Trophic differentation in Ilyodon, a genus of stream-dwelling goodeid fishes: speciation versus ecological polymorphism. Evolution. 34: 259-270. 